\title{
Virtual Hang-gliding over Rio de Janeiro
}

\author{
Luciano Soares, Leonardo Nomura, Marcio Cabral, Lucas Dulley, Marcelo Guimarães, Roseli Lopes, Marcelo Zuffo \\ Laboratório de Sistemas Integráveis - Departamento de Engenharia de Sistemas Eletrônicos \\ Escola Politécnica da Universidade de São Paulo - São Paulo - SP - Brazil \\ \{lsoares,nomura, mcabral,dulley,paiva,roseli,mkzuffo\}@lsi.usp.br
}

\begin{abstract}
Rio de Janeiro is famous for its tourist places like Corcovado and Sugarloaf. In this virtual tour the user can experience what a real hang-gliding flight feels like, with great navigability. The user has a stereoscopic visual and audio experience and wind sensation. In this application we are using cluster technology, which enables more graphical outputs besides distributing the tasks to achieve a better resource balance.
\end{abstract}

\section{Introduction}

Virtual tours are becoming very popular since the Internet letting users access far away content. Today it is possible to virtually visit cities, museums, exhibitions around the world. It is very common the use of $360^{\circ}$ cameras to enable the user to see an environment, but the use of virtual reality that brings more movement freedom is growing. Hang-gliding is a very enjoyable sport, provided in many tourist places, it is even possible two people hang-gliding. But many people are still afraid to fly in such system or unable to travel to Brazil. For this reason a virtual hang-gliding over Rio de Janeiro is important.

Rio de Janeiro is one of the most beautiful cities in Brazil and hang-gliding is a very common sport there. Figure 1 presents a simulation on a CAVE that shows the most important part of the city visualization. Using virtual reality we developed a virtual tour over Rio de Janeiro city. In this system the user can navigate over the city and see and hear its beautiful sights from a different perspective providing a safe and fun environment where people can experience a truly immersive hang-gliding. By easing fear and eliminating the risks, the experience is enhanced. It could cure problems caused by lack of selfconfidence. And it is a tool for training as well. This solution could even encourage people to try a real hanggliding experience.

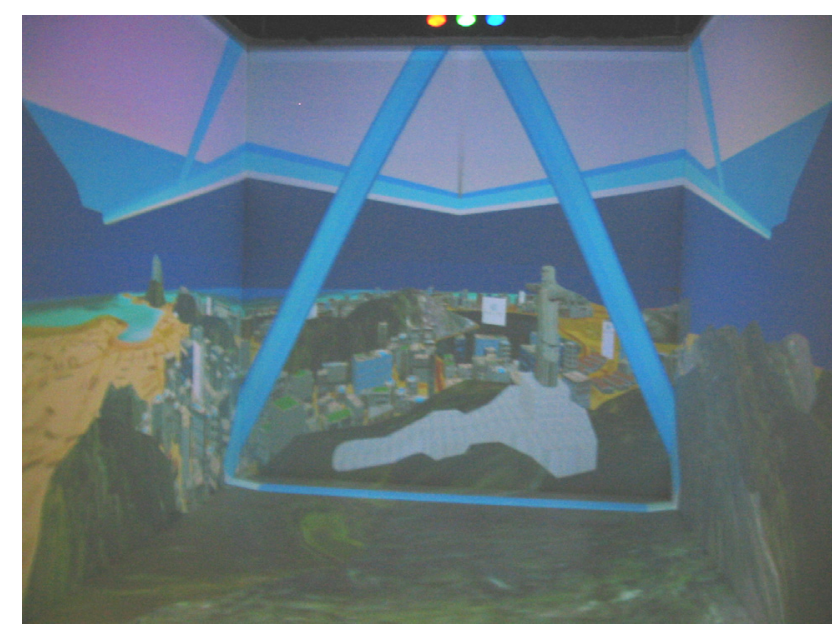

Figure 1 - View of Rio de Janeiro

This virtual tour is composed by a real hang-glider structure of about 9 square meters, where the user can rest on a hang strap. Usually hang gliders are controlled by shifting the pilot's weight with respect to the glider. In our case we are using a modified joystick connected to the glider's frame. It is very easy to control in just few seconds the user is able to control the movement of the glider.

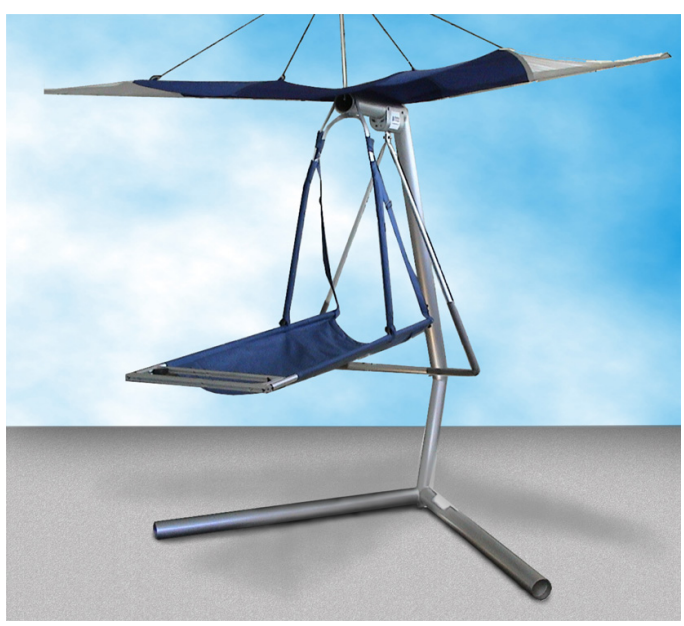

Figure 2 - hang-gliding model 
For user visualization we are using I-glasses SVG 3D HMD which supports stereoscopic vision and audio stereo, see Figure 3, this HMD was not intended for a massive use, but it works fine even in exhibitions with many people using the system. For head tracking we are using an Intertrax 2 tracker, see Figure 4, it has 3 degrees of freedom, enough to the user to rotate his head anywhere. The wind sensation is created by a fan positioned in front of the user, see Figure 5. This is a controlled fan that reacts when the user interacts with the glider. If the glider goes faster the speed of the fan is increased.

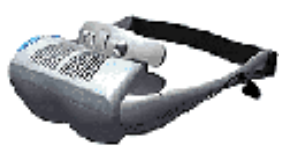

Figure 3 HMD

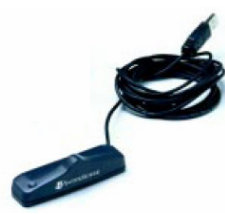

Figure 4

Head-tracker

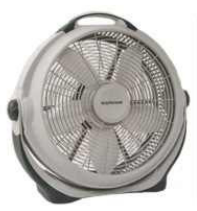

Figure 5

Electrical Fan

\section{Background and related work}

Dreamality Technologies [1] developed its Dream Glider simulation with input from the US Hang Gliding Association, the US Ultralight Association and Quest Air Soaring Inc. It includes a scoring system that rewards pilots with points. Other solution comes from the Tek Flight [2], their flight simulator is designed to aid new pilots to achieve comfort flying a hang glider in dynamic situations.

Another important project is the OpenSceneGraph [3]. It is a graphics toolkit for the development of high performance graphics applications, providing an object orientated framework on top of OpenGL, The project was started porting a hang gliding simulator written on top of the Performer scene graph running on IRIX. This project resulted in a company for hang gliding simulation [4].

The problem with these products is that they use just a simple monitor to enable the user to see what is going on. In our solution we use a HMD, that enables a better field of view, and with the resource of head tracking it is possible to simulate the head movement of the user, creating a more immersive simulation.

\section{Hang-gliding}

Hang-gliding is a sport that consists on flying, hanggliders are controlled by shifting the pilot's weight with respect to the glider. Pilots are suspended from a hang strap connected to the glider's frame. By moving forward and backward and side to side at the end of this hang strap, the pilot alters the center of gravity of the glider. This then causes the glider to pitch or roll in the direction of the pilot's motion and thus allows both speed control and turning. It can get a final seed speed about $130 \mathrm{~km} / \mathrm{h}$ and stay flying during many hours.

\section{Development}

The model of Rio de Janeiro was created as part of a project for a tourism exhibition. It was created with Alias|wavefront Maya in just 1 month due to time constraints, regarding the exhibition. Figure 6 presents a snapshot of the modeling window where it is possible to see the Corcovado Christ and Sugar loaf. The model is saved in VRML and then converted to X3D [5], it consists of 18.542 polygons. Also was created an avatar of the glider because the user is wearing a HMD and can not see the real one. The model does not constraint the proportions of the real world, we decided to that because it creates a more fun travel in a short period.

Some modifications are still necessary for the converted animations, which are based on interpolation nodes, which change its position during the time. For instance the cable car, that goes up and down between the two mountains. Some textures where create, like the grass and plants over the mountains, and other textures where get from the real place, like the Copacabana Palace Hotel, among others.

The sound simulation has the background music "The Girl from Ipanema" [6], and some points in the environment have special sounds, like in Maracanã, the biggest soccer stadium worldwide, and Sambódromo, the carnival parade avenue. The head tracking is used to enable the user to rotate his head and see and hear different scenes at any time. These sounds are just $\mathrm{mp} 3$ sound files that are easily read for the environment.

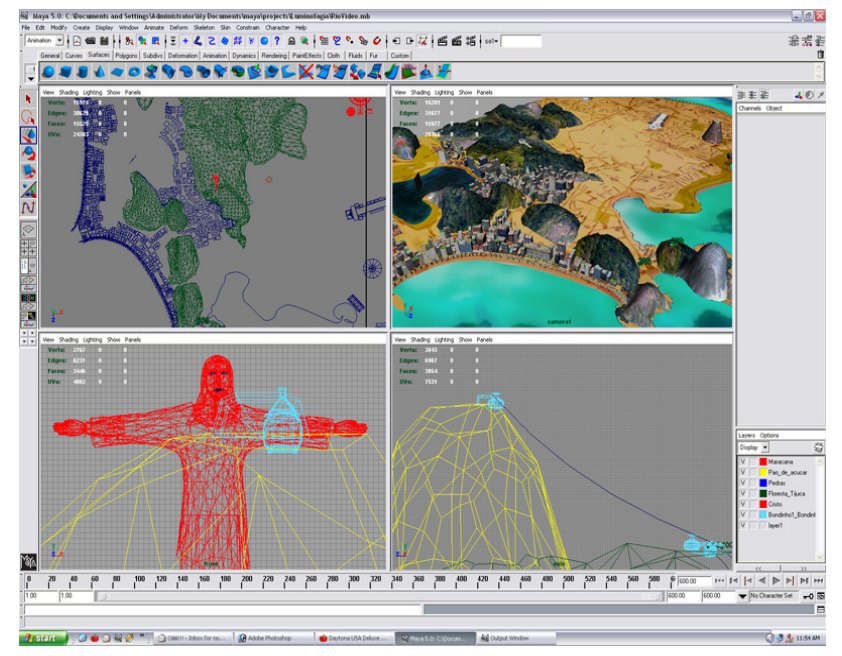

Figure 6 - Maya tool editing Rio de Janeiro

The tool used to visualize is Jinx [7]. Jinx is a tool developed internally at the University. It is a 3D browser and supports X3D files. Jinx was projected to support 
simulations using commodity cluster technologies. This way each computer is responsible for some specific tasks. It supports stereoscopic graphics and 3D sound, besides other possible expansions.

The X3D system is based on a scene graph, because of the architecture of $\mathrm{C}++$, it is very simple to simulate each $\mathrm{X} 3 \mathrm{D}$ node as an object. The X3D system has some nonlinear links. A XML parser is necessary to parse an X3D file, as well as the configuration files for Jinx, which are also in XML format. For this we developed our own parser.

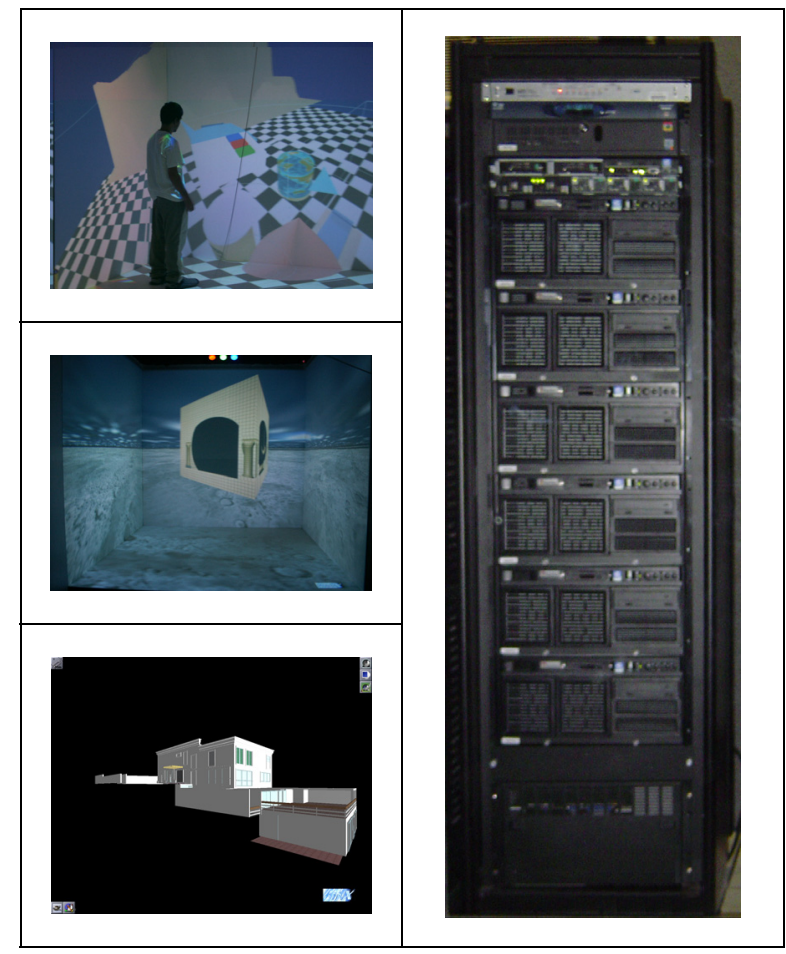

Figure 7 - Some images from JINX and Cluster

Figure 7 presents some simulations used with Jinx and the cluster. This cluster is capable to drive until 6 stereo screens and audio channels. In our solution we have also a 64 bit computer to calculate the simulations, like collision detection, one computer for console management, and another one for video access and one KVM.

\section{Results}

The ride is a user-controlled flight through Rio de Janeiro's skies. In this virtual world the user can look in any direction, appreciate the most famous sights of the city including Sugarloaf and Corcovado in addition feeling the blow of the wind in user's face.

The system was already presented in an exhibition in a tourist conference in Brazil, and it was a success. The
Figure 8 shows a user interacting with the solution in the exhibition.

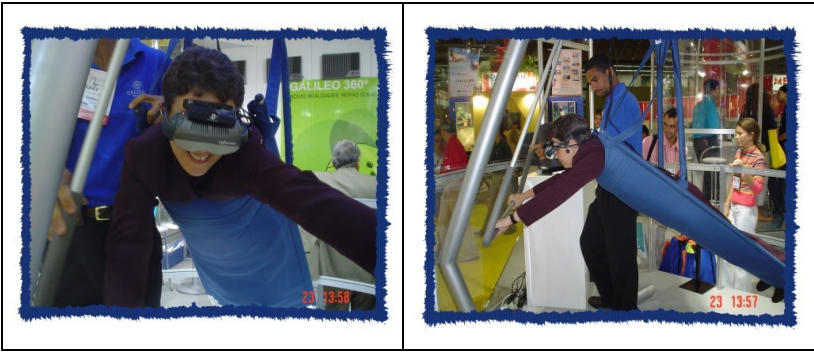

Figure 8 - Hang-gliding in use

Figure 9 shows the images that the user sees. This simulation was produced by a single Linux computer. There is a clock at the upper right corner, this clock was used in the exhibition to control the time the user can stay at the glider. He can see the time decreasing during the flight, and also a sound alarm.
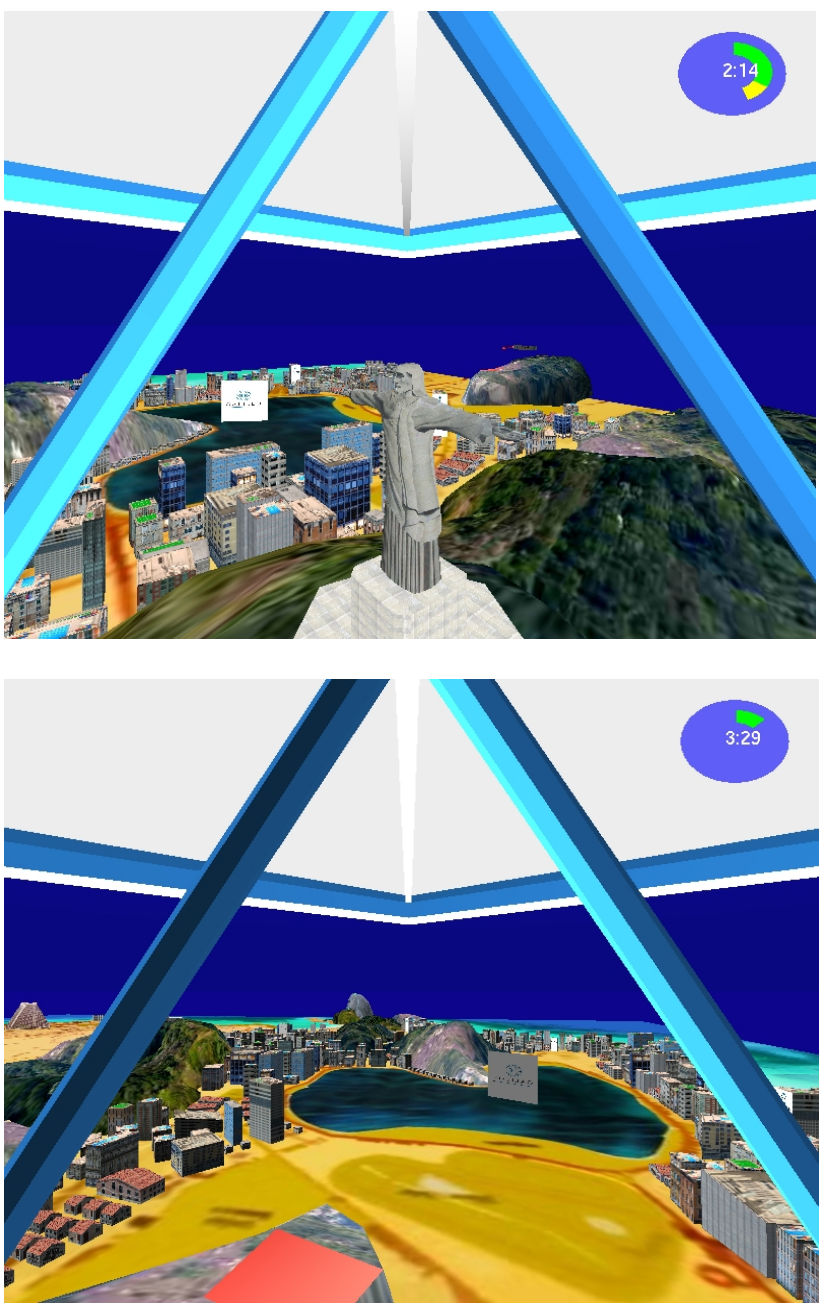

Figure 9 - Hang-gliding images 


\section{Conclusion and Future Work}

Figure 10 presents the model of the new prototype in development. In this new solution will have a large screen projection and external speaker, enabling the public to see and hear what is going on. Besides we are going to use two electrical fans for more precise wind sensation.

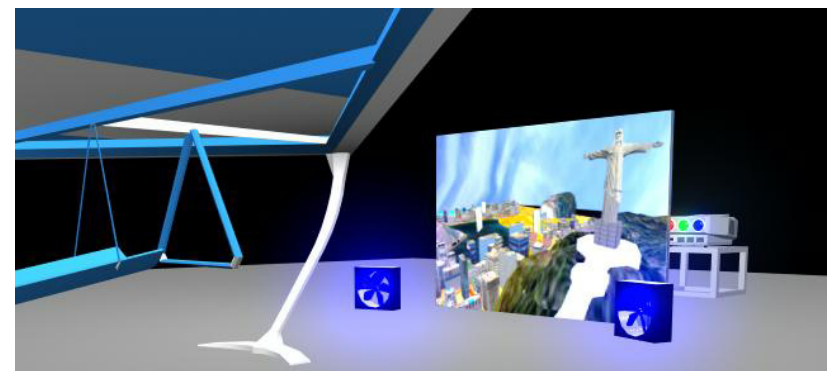

Figure 10 - New prototype

Another alternative is the use of domes (spherical screens) as in Figure 11. The glider, in this case, would be in the center of the dome, providing a large field of view to the user, enhancing the sense of presence. We need to do some tests, but probably this system will not need an $\mathrm{HMD}$, and probably the quality will be the same.
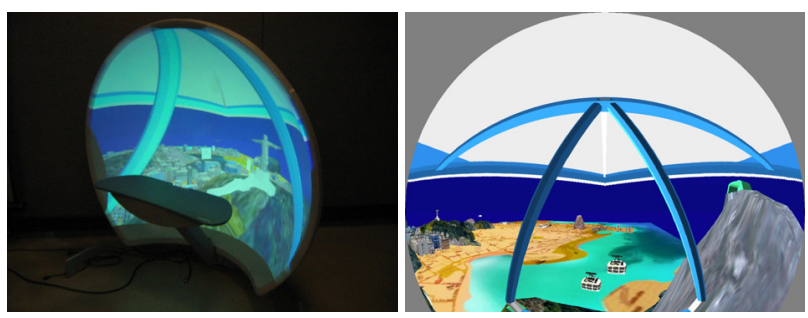

Figure 11 - Spherical Projection

Finally we just became to get some aerial photos, like Figure 12, and use as terrain, given a better reality. The use of wind sound feedback is also in our scope.

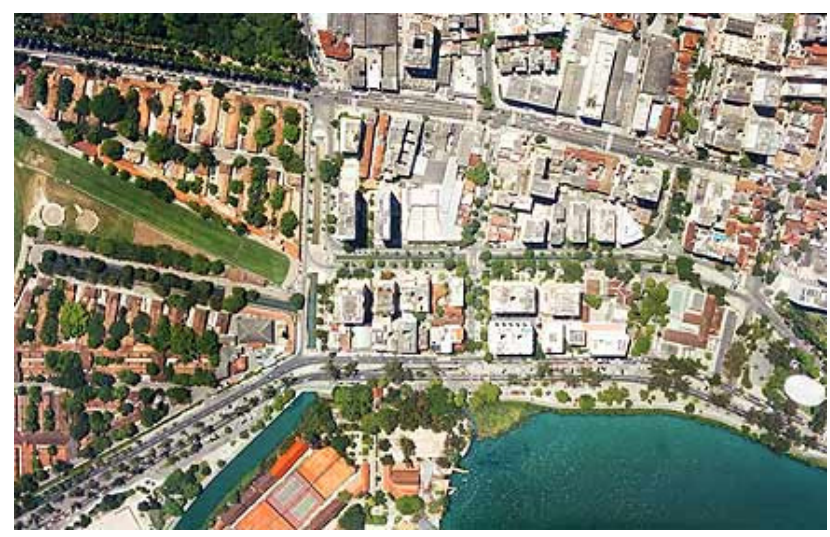

Figure 12 - Aerial photo

\section{Acknowledgements}

We would like to specially tank Prof. João Antonio Zuffo, for his constant support and advising. This project was sponsored by Galileo do Brasil and Absolut Technologies, and all the laboratory infrastructure was funded by FINEP Financiadora de Estudos e Projetos: SISCOMPRO project grant 01.02.0288.00.

\section{References}

[1] Dreamality Technologies, available at: http://www.dreamalitytechnologies.com/

[2] Tek Flight, available at: http://www.tekflight.com

[3] Open Scene Graph, available at: http://openscenegraph.sourceforge.net/introduction/

[4] Ander Engineering, available at: http://www.andesengineering.com/

[5] X3D ISO/IEC FCD 19775:200x, Information technology -- Computer graphics and image processing -- Extensible 3D (X3D), Available at http://www.web3d.org.

[6] Moraes, V., Jobim, T. Garota de Ipanema, Brazilian song, 1962

[7] Kaczmarski, H., Zuffo, M. K., Schaeffer, B., Goudeseune, C., Soares, L. P., Introduction to Commodity Clusters for Multiprojector Displays, Tutorial of Workshop on Commodity Clusters for Virtual Reality IEEE VR 2003 